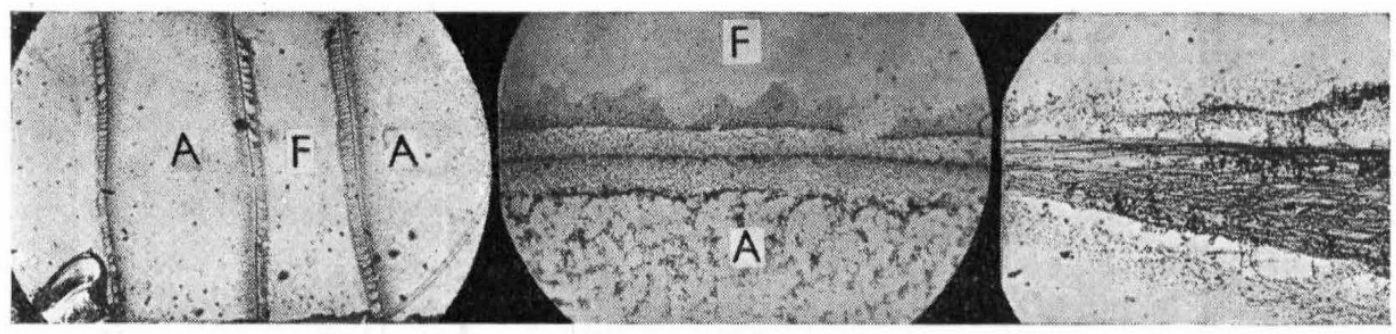

1. A DRIED FILM ( $F)$ OF SODIUN THYMONUOLEATE DISSOLVED IN EG ALBUMEN ON WHIOH LINES OF ALKAL UREA SOLUTION (A) WERE DRAWN $\begin{array}{lll}\text { ARTEFACTS } & \text { RESEMBLING } & \text { LAMPBRUSH } \\ \text { STRUCTURES } & \text { ARF FORMED, }\end{array}$ CARMINE STALN.
2. THREE IINES OF CONDENSATION OF STAINABLE GRANULES FORMED ON THE EDGE OF A DROP $(A)$ OF ALKALI UREA SOLUTION DEPOSITED ON A DRY FILM $(F)$ OF SODIUM THYMONUCLEATE DISSOLVED IN EGG ALBUMEN. ACETO-
3. MigRATION OF STAINABIE GRANULES AND FOBMATION OF CONDENSATION LINE. A BONDLE OF FIBRES DOLUECTD FROM THE SURFACE OF IN KALI UREA SOLUTION. ACETOCARMINE.
When a drop of alkali urea is deposited on a dry fllm of sodium thymonucleate dissolved in egg albumen, one or several such con densation lines may be formed.

The solution of sodium thymonucleate in egg albumen is capable of forming an organized layer on the surface. This layer when removed on a glass rod, like a folded umbrell is seen even without staining to consist of show th wavy flbres. These fibres treated with alkali urea solution sbove the same behaviour of the stainable granules as is described urea ; they emigrate from the fibres into the surrounding alkal of the flbres and at a certain distance from the fibre a condensation line is formed parallel to it. The above experiments on the migration of stainable granules seem in indicate that the appearance of longitudinal strings of condensation not, without reservation, be used as an evidence showing that these two condensation strings originally existed in the salivary gland chromosomes.

Department of Biology and Genetics, B. M. SuIZYNskz. Polish Medical School,

and Institute of Animal Genetics,

University of Edinburgh. Oct. 25.

'Calvin, M., Kodani, M., and Goldschmidt, R., Proc. Nat. Acad. Sci. 26, $340(1940)$.

Kodani, M., J. Hered., 32, 146 (1941).

'Kodani, M., J. Hered., 33, 114 (1942).

\section{Interaction Between Regenerating Limbs}

Data obtained elsewhere ${ }^{1}$ appeared to show evidence of competitive interaction between a pair of regenerating Crustacean limbs: the effect was not statistically significant, however. Specific experiments to test this have also failed to yield a signiflcant result. In forty individuals of Asellus aquaticus (L.) both limbs of the seventh and one of the eighth thoracic pair (right and left in equal numbers) were amputated at the autotomy plane. The regenerating seventh pair were measured when first visible externally, that is, after regeneration for one instar. The limb on the same side as the regenerating limb of the eighth pair showed a mean excess of 2.6 per cent in length over its partner, but showed a mean excess of 2.6 per cent in length over its partner, but
with a probable error of 1.2 per cent. The effect is little greater than might be obtained by chance: in a random sample of forty ndividuals, with only the two limbs of the geventh pair regenerating, the left limb showed a mean excess of $1.7 \pm 1 \cdot 1$ per cent over the right (while in a previous sample ${ }^{2}$ an excess of the right over the left had seemed probable).

Paulain $^{3}$ also observed an insignificant competitive effect between regenerating limbs of Arthropods; the data of von Ubisch ${ }^{4}$ showing mutual stimulation between such limbs do not appear to have been tested statistically. Interaction would seem to be, at the most, slight, and variable in character. Recent work ${ }^{3,5,6}$ indicates that interaction between a regenerating and a non-regenerating limb is equally slight.

Department of Physiology, A. E. NEEDHAM.

University, Manchester, 13.

${ }^{1}$ Needham, A. E., J. Exp. Biol., 21, 144 (1945).

'Neodham, A. E., Proc. Zool. Soc. Lond., A, 113, 44 (1943).

'Paulain, R., Proc. Zool. Soc. Lond., A, 108, 297 (1938)

'von Ubisch, L., Arch. Ent. Mech. Org., 41, 237 (1915).

'Tazelaar, M. A., Proc. Zool. Soc. Lond., A, 108, 257 (1938).
'Dixey. L. R., Proc. Zool. Soc. Lond., A, 108, 289 (1938).

\section{A New Electronic Band-System of $\mathrm{BaCl}$}

WE have recently had occasion to photograph the emission spectra of the flames from various pyrotechnic compositions containing barium and chlorine compounds. The well-known green and ultraviolet band-systems of $\mathrm{BaCl}$ are, of course, strongly developed in these
sources, but in addition, a number of bands in the region $7800-9500 \mathrm{~A}$. sources, but in addition, a number of bands in the region $7800-9500 \mathrm{~A}$
sre present on our spectrograms. These bands do not appear to have are present on our spectrograms. These bands do not appear to have oystem of $\mathrm{BaCl}$

The spectrograms were taken in a flrst order of a $2.4 \mathrm{~m}$. grating in an Eagle mounting (linear dispersion about $7 \cdot 25 \mathrm{~A} . / \mathrm{mm}$.) ? they show a number of rather close sequences of apparently double-headed bands degraded to longer wave-lengths. The violet edges of the two strongest sequences are at 8420.8 and $9098.0 \mathrm{~A}$. About fifty-five bands have been arranged into a Deslandres vibrational scheme with the 8421 band as 0,0 band, while some twenty-five bands have been assigned to an array involving the 9098 band as 0,0 band. The data the second heads lie at about $4.1 \mathrm{~cm} .^{-1}$ to the violet.

$\begin{array}{ccccl} & \nu_{e} & & \omega_{e} & x_{e} \omega_{e} \\ b & 11880 \cdot 0 & & 255 \cdot 25 & 0 \cdot 83 \\ a & 10995 \cdot 3 & & 256 \cdot 35 & 0 \cdot 73 \\ X^{2} \Sigma & 0 & (b) & 279 \cdot 4 & 0.765 \\ & & (a) & 279.5 & 0.755\end{array}$

The above values of $\omega_{e}{ }^{\prime \prime}$ and of $x_{e}{ }^{\prime \prime} \omega_{e}{ }^{\prime \prime}$ are in satisfactory agreement with those for the ground-state of $\mathrm{BaCl}$ determined by Parker ${ }^{1} \mathrm{from}^{-1}$ an analysis of the green system, namely, $278 \cdot 4,280.5$ and $0.80 \mathrm{~cm}^{-1}$. The closeness of the bands within a given sequence makes it difficult to observe the vibrational isotope effect due to $\mathrm{Ba}^{35} \mathrm{Cl}, \mathrm{Ba}^{87} \mathrm{Cl}$, but for several favourably placed bands, notably the earlier members of the more refrangible 0,1 sequence, heads due to the heavier isotopic molecule have been identifled with some certainty.

The evidence that the carrier of the new bands is $\mathrm{BaCl}$ is therefore very strong. Perhaps the most likely interpretation of the results is that the transition involved is $8 \Pi \rightarrow X^{2} \Sigma$ the doublet spacing of the upper state being about $885 \mathrm{~cm}^{-1}$. It is hoped to settle this point at a later date by extending the observations to longer wave-lengths. R. F. BARROW.

Physical Chemistry Laboratory,

University of Oxford.
Und Nov. 7.

1 Parker, A. E., Phys. Rev., 46, 301 (1934).

\section{Ammonia Spectrum in the $1 \mathrm{~cm}$. Wave-length Region}

IN 1933 Cleeton and Williams ${ }^{1}$ observed a broad absorption band in mmonia at atmospheric pressure in the region of $1 \mathrm{~cm}$. wave-length this is attributed to the 'inversion' of the pyramidal ammonia molecule which occurs when the nitrogen atom swings through the plane of the three hydrogen atoms. We have re-examined this phenomenon using a new technique, and have found that, as the pressure is reduced from $600 \mathrm{~mm}$. mercury to $0.2 \mathrm{~mm}$, so that the frequency of the collisions
becomes small compared with the frequency of the radiation, an laborate system of absorption lines appears.

The absorption coefficient was determined at high pressure by measuring the decrease in the power transmitted through a length of wave-guide into which the gas could be introduced. The results were in substantial agreement with those of Cleeton and Williams. At pressures below $10 \mathrm{~mm}$. the absorption coefficient was derived from observations of the damping caused in a resonant cavity by admission of the gas ; in this way absorptions as small as $2 \times 10^{-6} \mathrm{per}$ cm., which otherwise wou

The accompanying reproduction shows the spectrum observed at a pressure of $1.2 \mathrm{~mm}$. mercury; owing to the low collision frequency numerous lines may be distinguished, their breadth being about these lines have been resolved.

The extensive fine structure shown is due to the centrifugal distortion of the molecules in the various rotational levels; Hsi-yin Sheng, Barker and Dennison ${ }^{2}$, using a potential energy curve proposed by Manning ${ }^{3}$, have derived a formula for the fine structure of the inversion frequency of the form

$$
\nu=\nu_{0}-A\left(J^{2}+J\right)+B K^{2},
$$

$J$ and $K$ being the rotational quantum numbers. The frequencies of the lines observed by us are accurately represented by a formula of the same type with the addition of a second-order term from the expansion of an exponential, namely,

$v=0.7940-0.0050_{3}\left(J^{2}+J\right)+0.0070_{4} K^{2}$
$+0.63\left\{-0.0050\left(J^{2}+J\right)+0.0070\right.$

$$
\begin{aligned}
& 0-0.0050_{5}\left(J^{2}+J\right)+0.0070_{4} K^{2} \\
& +0.63\left\{-0.0050\left(J^{2}+J\right)+0.0070 K^{2}\right\}^{2} \mathrm{~cm} \cdot .^{-1} \text { (vacuo). }
\end{aligned}
$$

Eighteen lines have been examined at a pressure of $0.5 \mathrm{~mm}$. Their intensities agree to within 5 per cent with those calculated from the statistical weights and transition probabilities, thus confirming the 